\title{
Agenesis of Inferior Vena cava in complicated by recurrent Deep venous thrombosis and Pulmonary embolism in a tertiary hospital, Sudan
}

\author{
Mohammed Elfaki ${ }^{1}$, Asma Abdallah ${ }^{1}$, and Samoal Daffalla ${ }^{1}$ \\ ${ }^{1}$ Ahmed Gasim Cardiac Surgery and Renal Transplantation Center
}

June 9, 2020

\begin{abstract}
A 47 year-old man who was successfully treated for PE . later on Discovered to have left sided venous engorgement in the abdomen and left lower limb. He was later discovered to have congenital absence of inferior vena cava and was prepared for investigations including venography and coagulation profile
\end{abstract}

Key Clinical Message : Congenital absence of Inferior Vena cava is a rare anomaly . As the case for other congenital anomalies, it should be suspected at all ages in any patient who presents with recurrent complications of venous stagnation such as PE and recurrent DVTs. Key Clinical findings are : recurrent DVT and/or PE , Stigmata of Venous engorgement as a result of development/enlargement of collateral veins .

Background: Congenital absence of Inferior Vena Cava ( CAIVC) is a rare anomaly in which the lower-body venous return is impaired. There is usually engorgement of other veins especially Azygos and Hemiazygos systems.

Ethical Clearance : A full Informed consent was taken from the patient. Permission was taken from the patient for publication.

Case summary : a 47 year-old man presented who was successful treated for PE . Later discovered to have left sided venous engorgement in the abdomen and left lower limb. He was later discovered to have congenital absence of inferior vena cava and was prepared for further investigations including venography and coagulation profile .

Conclusion: CAIVC could present later in life with complications as a result of Blood stagnation including DVT and PE .

Introduction: Agenesis of the inferior vena cava (IVC) is a rare abnormality that occurs in less than $1 \%$ of the population. The origin of the inferior vena cava is a complex process that occurs between the sixth and eighth week of gestation. When the process of formation of the vena cava incompletely occurs or it does not occur, we have a compensatory dilatation of the azygos system to help the venous drainage of the lower segment of the body. $(1,2)$

Case presentation :

a 47-year-old male come to our clinic in 07/02/2019 complaining of,chest pain, shortness of breath and cough for the previous 5 days. The Chest pain was retrosternal, sudden in onset and was not radiated or referred 
. In addition, the pain was severe, dull in character and there were no reported exacerbating factors . It was associated with shortness of breath at rest and cough. On examination, the patient was ill- looking, dyspneic with a respiratory rate of 30 . He was pale, and axillary temperature was 38.1 . Blood pressure was 110/73 and the Mean Arterial Blood Pressure (MAP) was 82 . Finally, SpO2 was $90 \%$ on room air improving to $94 \%$ with high-flow oxygen via mask .

Regarding cardiovascular examination, there was pulsations in the neck which was found to be an arterial pulsation. JVP was not raised. Pulse was 139 , sinus rhythm, good volume, normal character and there was no radio-radial or radio-femoral delay. Precordium examination was normal . Chest examination was also normal apart from some transmitted sounds and possible fine crackles over lower zones . Abdominal examination revealed tortuous, snake-like protrusions extending from the epigastrium down about 2-3 cm from the midline in the left side radiating away from the umbilicus . In addition, there are few scattered swellings in the left lumbar and the left iliac regions. ( Figure 1 ). These swellings were found to be engorged superficial veins. Otherwise, the rest of abdominal examination was unremarkable .

On Examination of the lower limbs, the left lower limb was swollen up to the knee and it was darker in color as compared to the other limb. Also, there were multiple crustations and scratch marks more prominent on the medial ankle extending medially just below the knee but there were no ulcers. The limb was pitting with normal temperature . Later on, a diagnosis of DVT was confirmed using Doppler U/S scan . The right lower limb was completely normal .

After that, a set of Investigations were requested . CTPA confirmed a diagnosis of pulmonary embolism ( PE ). CXR was normal. CT chest revealed dilated thrombosed azygos veins, subpleural honeycombing, early traction bronchiectasis, reticular interstitial thickening and lung architectural distortion . (Figure 2) . CT angiography for the lower limbs was completely normal . The patient has a previous venography that was done many years ago but unfortunately, it was not available at the moment. It showed complete absence of inferior vena cava and dilated azygous and hemi-zygous venous systems more in the right side . This finding was also noted in the abdominal CT ( Figure 3 )

BNP was high a level of $700 \mathrm{pg} / \mathrm{mL}$. He received fractionated heparin $60 \mathrm{mg}$ and warfarin $5 \mathrm{mg}$ was thereafter continued in addition to analegesia. The patient's condition was improved, SOB and chest pain disappeared and repeated BNP was normal at a level of $10 \mathrm{pg} / \mathrm{ml}$. The patient has had several episodes like this before and he was admitted at least once to the hospital for treatment of Pulmonary embolism . The diagnosis is Congenital absence of Inferior vena cava complicated by recurrent lower limb DVT and PE . Further investigations were requested to rule out other causes of VTE including Coagulation factors . Unfortunately , we could not be able to view the results of these tests because the patient did not bring them back later .

Author Contribution: Mohammed I Alfaki and Asma Abdallah : Were involved in selection of the case. In addition, they worked together in writing and editing the manuscript. Samoal Dafallah : He supervised the case presentation and management. He guided the process of selection and involved in final approval of the manuscript . All authors were involved in the editing and final approval of the manuscript.

References :

1. Phillips E.Embryology, normal anatomy, and anomalies Ferris E.J., Hipona F.A., Kahn P.C., Phillips E., Shapiro J.H. (Eds.), Venography of the inferior vena cava and its branches, Williams 8 Wilkins, Baltimore (1969), pp. 1-32 .

2. Leg Ulceration in Chronic Venous Insufficiency Caused by an Absent Inferior Vena Cava Hiroo Amano, Yayoi Nagai, Yukie Endo, Tomoko Iwasaki and Osamu Ishikawa Department of Dermatology, Gunma University Graduate School of Medicine, Maebashi, Gunma, Japan

3. Arrazola L, Long A, Moss A, Sozen H, Najarian J. An absent inferior vena cava in a pediatric renal transplant recipient. Clin Transplant 2000; 14: 360-362. 4. Dougherty MJ, Calligaro KD, DeLaurentis DA. Congenitally absent inferior vena cava presenting in adulthood with venous stasis and ulceration: a surgically treated case. J Vasc Surg 1996; 23: 141-146. 

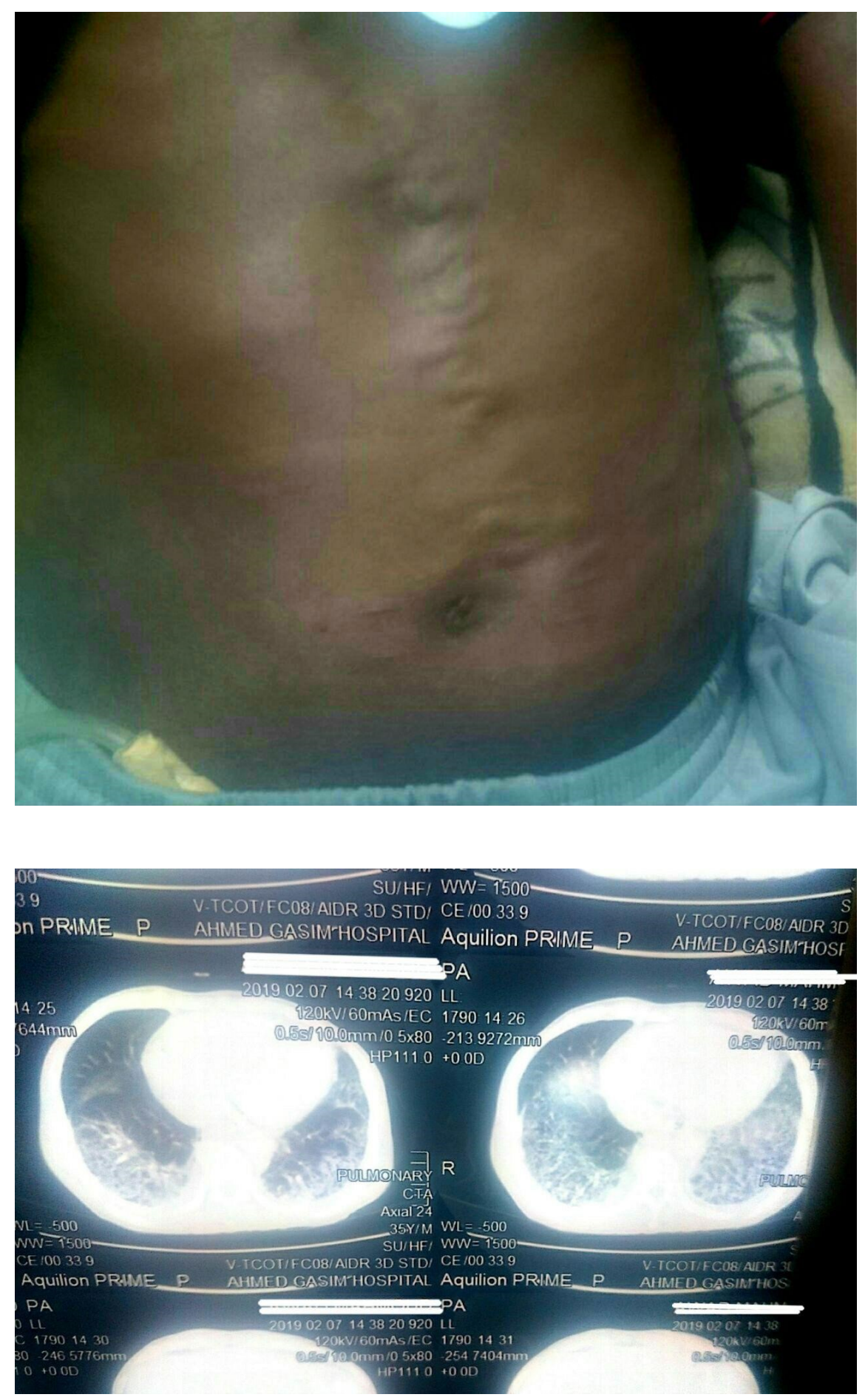

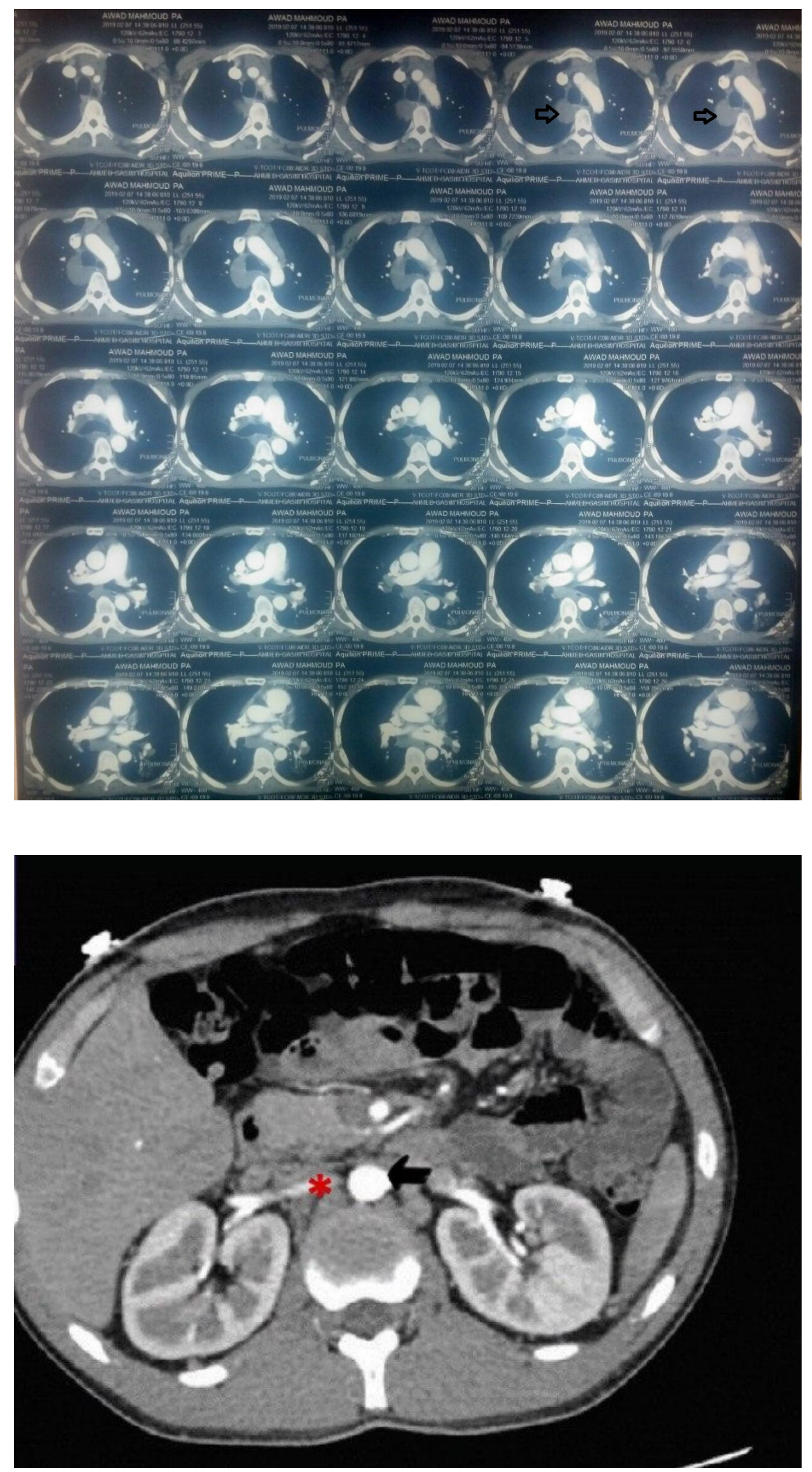